Results There were 405 genes, 3 splicing variants, and 2 promoters that were statistically significantly different between case and control. We detected abnormal thyroid function, impaired myelination, and delayed ossification of the mandible in the poor oral feeder $\left(10^{-5}\right.$ $<\mathrm{p}<10^{-2}$ ). Genes involved in neurodevelopment, hyperphagia, and adipocyte development were differentially expressed between subjects $\left(10^{-3}<\mathrm{p}<10^{-2}\right)$.

Conclusions Targeted comparative RNA sequencing analyses identify global, and patient specific, aberrations in developmental pathways directly related to oral feeding pathology. Our study demonstrates the feasibility of neonatal salivary sequencing for identifying key regulatory genes and pathways that are differentially expressed and regulated between successful and unsuccessful oral feeders, and suggests that this approach will lead to new insights into neonatal pathophysiology.

\section{THE DIURETIC EFFECT OF UREA ANALOG DIMETHYLTHIOUREA IN RATS}

doi:10.1136/archdischild-2012-302724.1415

1,20 Cil, ${ }^{2} \mathrm{M}$ Ertunc, ${ }^{2} \mathrm{R}$ Onur. 'Department of Pediatrics; ${ }^{2}$ Department of Pharmacology, Hacettepe University Faculty of Medicine, Ankara, Turkey

Background and Aims Urea plays important roles in urinary concentrating mechanisms in the kidney by contributing greatly in generation of hyperosmolar medulla, due to presence of urea transporters which mediate facilitated transport of urea. Urea transporter knock-out mice were reported to have increased daily urine volume. In this study, we investigated the possible diuretic effect of a urea analog and urea transporter inhibitor, dimethythiourea (DMTU), in rats.

Methods Female Wistar rats were divided into two groups, group 1 (Control, $n=7$ ) rats were injected with saline (ip), whereas group 2 (DMTU, n=7) rats were injected with $500 \mathrm{mg} / \mathrm{kg}$ DMTU (ip) and an additional dose of $125 \mathrm{mg} / \mathrm{kg}$ DMTU $8 \mathrm{~h}$ after.

Results DMTU administration induced a $\sim 3$ times increase in daily urine volume $(p<0.001)$ and decreased urine osmolality to \%35 of controls $(p<0.0001)$. DMTU also increased free water clearance $(p<0.0001)$ without a significant change in osmolar clearance. DMTU treatment caused an increase in urea clearance $(p<0.05)$ and fractional excretion of urea $(p<0.05)$ with a decrease in serum urea concentration $(p<0.001)$. DMTU had no effect on creatinine clearance or serum electrolytes, creatinine levels and osmolality.

Conclusions We report for the first time that DMTU has a prominent diuretic effect with increased urea excretion, which may be explained by the inhibitory effect of the drug on urea transporters. Our findings suggest that DMTU may be used as a diuretic agent and also could be used as a lead compound for development of a novel group of diuretics.

\section{INSULIN RESISTANCE CORRELATES IN OBESE ADOLESCENTS}

doi:10.1136/archdischild-2012-302724.1416

I Papandreou, G Papadopoulos, M Bourneli, Z Gerle. Paediatrics, Tzaneio General Hospital of Piraeus, Piraeus, Greece

Background and Aims Obesity is an epidemic that affects adolescents and afflicts changes in their metabolic profile. The purpose of our study was to compare glucose metabolism, insulin resistance, dyslipidemia and blood pressure between obese and healthy adolescents.

Methods We included in our study 126 adolescents followed at the outpatient clinic of our hospital, aged 13.9 \pm 2.01 (Mean \pm SD) in the course of 2011. The Body Mass Index (BMI) was calculated from weight and height measurements and was used to divide the adolescents into two groups, (obese, those above the 95th percentile, which corresponds to a BMI of 30 (considered obese in adults) and non obese). Blood pressure, fasting insulin, glucose and lipid blood levels were measured. Estimates of insulin resistance (homeostatic model assessment (HOMA-IR) and the quantitative insulin sensitivity check index (QUICKI), were derived from fasting measurements. For the statistical analysis we used SPSS 20.0 (IBM Corp.). Mann-Whitney and Spearman tests were applied.

Results Among the adolescents in our study 47 were obese. Obese adolescents had a higher systolic and diastolic blood pressure ( $p<0.001$ and p: 0.04 respectively), higher blood levels of fasting insulin $(p<0.001)$ and lower High Density Lipoprotein(HDL) (p:0.01) compared to non-obese. Insulin resistance and insulin sensitivity indexes were associated with obesity (HOMA-IR, $\mathrm{p}<0.001$, QUICKI $\mathrm{p}<0.001$ ).

Conclusions Increased insulin resistance, higher blood pressure and low levels of HDL were associated with increased adiposity among adolescents. It is therefore necessary to screen for elevated blood pressure and hyperlipidaemia amongst obese adolescents.

\section{METABOLIC SYNDROME AND INSULIN RESISTANCE IN CHILDREN AND ADOLESCENTS}

doi:10.1136/archdischild-2012-302724.1417

${ }^{1} \mathrm{M}$ Erol, ${ }^{1} \mathrm{O}$ Yigit, ${ }^{2} \mathrm{M}$ Çömçe, ${ }^{1 S}$ Zengi, ${ }^{3} \mathrm{O}$ Zengi. ${ }^{\text {PPediatrics; }}{ }^{2}$ Family ph; ${ }^{3}$ Department of Biochemistry, Bagcllar Training and Research Hospital, Istanbul, Turkey

Overweight and obesity in children and adolescents have become a major public health problem in recent years. The prevalence of metabolic syndrome in childhood increases in parallel with the high prevalence of obesity in children. The metabolic syndrome was defined as having at least three of the following: abdominal obesity, low high-density lipoprotein (HDL) cholesterol, hypertriglyceridemia, hypertension, and/or impaired glucose tolerance. Insulin resistance is the principal metabolic abnormality that is common to the development of the metabolic syndrome in children and adults.

Metabolic syndrome was found in \%17.2 (12 cases). In our study, we aimed to investigate the potential risk factors in development of obesity evaluate metabolic syndrome and insulin resistance frequency in children and adolescent population. Seventy obese children with a mean age of $10.8 \pm 2.47$ years and body mass index $>$ 95 th percentile were enrolled the study. Patients were assesed birth weight, duration of breast-feeding, prevalence of obesity and typ 2 diabetes in parents, age at onset of obesity and components of metabolic syndrome. The diagnosis of metabolic syndrome were defined according to modified WHO criteria adapted for children. Each subject was submitted to an oral glucose tolerance test. Obesity and type 2 diabetes rates in parents of cases, were \%42.8(30 cases) and $\% 12.8$ (9 cases) respectively. According to homeostasis model assessment insulin resistance (HOMA-IR) index, insulin resistance was determined \%88.5 (66 cases). İn our study birth weight, duration of breast-feeding weren't assosiation with metabolic syndrome and insulin resistance.

\section{NUTRITIONAL SURVEILLANCE IN OVERWEIGHT/OBESE COELIAC CHILDREN (OCC)}

doi:10.1136/archdischild-2012-302724.1418

${ }^{1} \mathrm{R}$ Mercurio, 'D Guttadoro, ${ }^{2} \mathrm{~A}$ Piedimonte, ${ }^{1} \mathrm{~A}$ Mosca, 'L Petrarca, 'M Barbato, ${ }^{1}$ A Giacomini, 'A Vania. 'Dept of Paediatrics and Paediatric Neuro-Psychiatry; ${ }^{2}$ Institute of Nutrition, 'Sapienza' University of Rome, Rome, Italy

Background and Aims Gluten-free-diet (GFD) in Coeliac Disease is often complicated by excessive body weight. This study aims to evaluate the efficacy of a balanced GFD.

Methods Retrospective study. We included OCC followed-up from 2006-2010, with a 1 year follow-up after the diagnosis. For all 